\title{
Atuação da Enfermagem na Atenção Primária à luz da prevenção do câncer de
}

\author{
mama \\ Nursing in Primary Care in the light of breast cancer prevention \\ La enfermería en Atención Primaria ante la prevención del cáncer de mama
}

Recebido: 29/11/2021 | Revisado: 03/12/2021 | Aceito: 10/12/2021 | Publicado: 18/12/2021

Maria Luiza Rêgo Bezerra

ORCID: https://orcid.org/0000-0002-3336-7760 Universidade Paulista, Brasil

E-mail: maria.bezerra@ docente.unip.br

Marcos Felipe Martins Mendonça

ORCID: https://orcid.org/0000-0002-3449-1157

Universidade Paulista, Brasil

E-mail: fellippedf@yahoo.com

\begin{abstract}
Resumo
Identificar as ações do enfermeiro na Atenção Primária em Saúde para a prevenção e detecção precoce do câncer de mama. Trata-se de revisão integrativa realizada em três bases de dados utilizando os descritores Enfermagem na Atenção Primária, Enfermagem oncológica e Neoplasias da mama, a partir destes ao final da busca obteve-se um número total de 15 artigos elegíveis. Ao longo dos anos o Sistema único de Saúde (SUS) tem caminhado positivamente melhorando a qualidade do cuidado e norteando o profissional Enfermeiro para uma melhor assistência em seu local de trabalho, exigindo desse habilidades como prover saúde desde o momento prevenção, diagnóstico, tratamento, reabilitação e cuidados paliativos. As tipologias destacadas foram Enfermagem na Atenção Primária à Saúde que é a atuação do Enfermeiro na porta de entrada do SUS e Prevenção do câncer de mama em UBS que é responsável pela qualidade de vida e longevidade da população adscrita. O combate ao câncer de mama realmente efetiva depende de muitos fatores, podendo destacar a atuação do Enfermeiro na prevenção e manutenção da saúde.

Palavras-chave: Enfermagem na atenção primária; Neoplasias da mama; Enfermagem oncológica.
\end{abstract}

\begin{abstract}
Identify the actions of nurses in Primary Health Care for the prevention and early detection of breast cancer. Integrative review conducted in three databases using the descriptors Nursing in Primary Care, Oncology Nursing, and Breast Neoplasms, from which, at the end of the search, a total number of 15 eligible articles were obtained.Over the years, the Single Health System (SUS) has walked positively, improving the quality of care and guiding the professional nurse for better care in the workplace, requiring skills such as providing health care from the moment of prevention, diagnosis, treatment, rehabilitation, and palliative care. The highlighted typologies were Nursing in Primary Health Care, which is the nurse's action at the SUS entrance door, and Breast Cancer Prevention in UBS, which is responsible for the quality of life and longevity of the enrolled population. The really effective fight against breast cancer depends on many factors, and it is worth mentioning the work of nurses in the prevention and maintenance of health.
\end{abstract}

Keywords: Primary care nursing; Breast neoplasms; Oncology nursing.

\section{Resumen}

Identificar las actuaciones de las enfermeras de Atención Primaria para la prevención y detección precoz del cáncer de mama. Revisión integradora realizada en tres bases de datos utilizando los descriptores Enfermería en Atención Primaria, Enfermería Oncológica y Neoplasias Mamarias, de los cuales, al final de la búsqueda, se obtuvo un número total de 15 artículos elegibles. A lo largo de los años, el Sistema Único de Salud (SUS) ha caminado positivamente, mejorando la calidad de la atención y orientando al profesional de enfermería para una mejor atención en el trabajo, requiriendo competencias como la prestación de cuidados de salud desde el momento de la prevención, diagnóstico, tratamiento, rehabilitación y cuidados paliativos. Las tipologías destacadas fueron Enfermería en la Atención Primaria de Salud, que es la acción de la enfermera en la puerta de entrada del SUS, y Prevención del Cáncer de Mama en la UBS, que es responsable de la calidad de vida y la longevidad de la población inscrita. La lucha realmente eficaz contra el cáncer de mama depende de muchos factores, y cabe destacar la labor de las enfermeras en la prevención y el mantenimiento de la salud.

Palabras clave: Enfermagem na atenção primária; Neoplasias da mama; Enfermagem oncológica. 


\section{Introdução}

Sendo o Câncer umas das doenças crônico-degenerativas com maior índice de mortalidade no Brasil e no mundo, e tendo destaque nas mulheres o Câncer de Mama, o movimento em prol da sua prevenção e detecção precoce teve início no Brasil nos anos 80 quando foi incluído no programa de Atenção Integral à Saúde da Mulher e ao longo dos anos o país vem buscando prevenir e tratar esse tipo de doença (INCA, 2020).

O Câncer de Mama se caracteriza como um problema de saúde pública que afeta mais de 100 países, já incluindo o Brasil nessa situação, porém cabe ressaltar também que não se tem somente o atendimento clínico do profissional enfermeiro e sua relação com a equipe e paciente para que essa situação seja controlada, outros fatores (dificuldade de acesso da população aos serviços públicos de saúde e dificuldade por parte dos gestores na logística dos diferentes níveis de atenção que precisam trabalhar em sintonia para prevenir e tratar precocemente esse tipo de Câncer podem e vão influenciar nesses indicadores (Silva et al., 2020)

Deste modo, no Brasil a expectativa para o triênio de 2020-2022 é que surjam cerca de 625 mil novos casos de Câncer no Brasil, sendo desse número uma média 66 mil casos de câncer de mama, números que são um alarme para a fomentação de programas que eduquem os profissionais enfermeiros na prevenção e detecção desse tipo de câncer nas mulheres (INCA, 2020).

Sendo essa atualmente a maior causa de incidência e letalidade de câncer entre as mulheres, justifica-se saber se o profissional atuante nas Unidades básicas de Saúde (UBS) tem conhecimento atualizado acerca da clínica que envolve o processo para prevenir e detectar precocemente o Câncer de Mama, isso inclui seus fatores de risco, métodos e exames para rastrear essa patologia, formulação de oficinas educativas para a comunidade e outros saberes (Ferreira et al, 2020).

Tendo dito, fica claro que com os programas de educação continuada aos profissionais enfermeiros e organização na linha de ação por partes destes otimiza o atendimento clínico através da detecção de possíveis anormalidades durante do acolhimento, aliados aos exames clínicos de rotina, educação às pacientes e solicitação de exames mais específicos quando necessário podem ser uma ferramenta de efetividade frente ao problema apresentado (INCA, 2020).

Sendo assim, questiona-se: Os profissionais enfermeiros na Atenção Primária à Saúde estão preparados para prevenir/ detectar precocemente o Câncer de Mama?

O crescente aumento do Câncer de mama ao longo dos anos faz com que seja necessário um olhar mais aprofundado acerca dos fatores que podem colaborar para a disseminação desse tipo de patologia na população, além de nos fornecerem um panorama sobre como está o nível de preparo dos profissionais da saúde que atuam na atenção básica.

Neste contexto o objetivo do estudo é compreender como as ações e práticas clínicas do profissional enfermeiro atuante nas Unidades Básicas de Saúde acerca da prevenção/ detecção precoce do câncer de mama e podem influenciar na saúde da mulher.

\section{Metodologia}

Este artigo trata-se de uma revisão de literatura integrativa, método que possibilita através da análise de literatura uma maior compreensão do estudo em questão e dá o devido do embasamento cientifico às práticas de Enfermagem (Sousa et al, 2018).

Foram adotadas a seguintes etapas para a elaboração do presente estudo: problematização embasada na situação atual dos enfermeiros atuantes em Unidades Básicas de Saúde; coleta, análise e interpretação dos dados; organização dos dados em categorias divididas em artigos que são voltados para a educação dos profissionais e artigos voltados para o cuidado ao paciente; Apresentação dos dados e considerações finais (Sousa et al, 2018). 
Com base nos descritores Enfermagem Oncológica, Neoplasias da Mama e Enfermagem na Atenção Primária, em outubro de 2021 foi realizada a busca nas seguintes bases de dados: Scientific Electronic Library Online (SciELO), Bases de Dados de Enfermagem (BDENF) e Literatura Latino-Americano e do Caribe em Ciências da Saúde (LILACS).

Foram adotados como métodos de inclusão artigos completos, publicados nos últimos cinco anos que estejam de acordo com os descritores selecionados, (2016-2021), sendo automaticamente excluídos aqueles que não estiveram em consonância com os descritores, que tenham uma data de publicação superior a cinco anos, artigos duplicados e artigos não disponibilizados de forma completa nas bases de dados.

Obteve-se na coleta de dados um total de 282, dos quais, 60 (21,28\%) foram encontrados na SciELO, 29 (10,28\%) na BDENF e $193(68,44 \%)$ na LILACS, artigos que inicialmente foram submetidos aos critérios de inclusão e exclusão previamente selecionados afim de filtrar o material científico e obter uma maior fidedignidade com o tema proposto, sendo que 35 artigos desse total se encontravam elegíveis para a próxima fase de seleção, sendo eles $10(28,57 \%)$ na base de dados da SciELO, 7 (20\%) na BDENF e 18 (51,43\%) na LILACS.

Numa análise seguinte dos 35 documentos foram selecionados através de leitura na íntegra $15(42,86 \%)$ artigos relevantes que estavam em conformidade com a questão da atuação da Enfermagem na Atenção primária na prevenção do câncer de mama, estes são 4 (40\%) da SciELO, 2 (28,57\%) na BDENF e 9 (50\%) na LILACS, o fluxograma detalhando essas etapas se apresenta a seguir no Quadro 1:

Quadro 1. Fluxograma da coleta e análise de dados.

\section{Estratégia de Busca}

\begin{tabular}{|cc|c|c|}
\hline \multicolumn{4}{|c|}{ Bases de dados e artigos encontrados } \\
\hline SCIELO & BDENF & LLACS \\
\hline 60 & & 29 & 193 \\
\hline \multicolumn{4}{|c|}{ Total de artigos encontrados: 282} \\
\hline
\end{tabular}

19 etapa de seleção

\begin{tabular}{|c|c|c|}
\hline SCIELO & BDENF & LILACS \\
\hline 10 & 07 & 18 \\
\hline
\end{tabular}

$2 \circ$ etapa de seleção

Total de artigos elegiveis para o estudo

\begin{tabular}{|c|c|c|}
\hline \multicolumn{3}{|c|}{ Total de artigos elegiveis para o estudo } \\
\hline SCIELO & BDENF & LILACS \\
\hline 4 & 2 & 9 \\
\hline
\end{tabular}

Fonte: Autores (Brasília-DF, 2021). 


\section{Resultados e Discussão}

Inicialmente são apresentados os resultados caracterizando os artigos do estudo (Quadro 2), logo após segue discussão e 2 categorias evidenciadas a partir dos resultados obtidos destes mesmos artigos, sendo elas: Enfermagem na Atenção Primária à Saúde e Prevenção do câncer de mama em Unidades Básicas de Saúde.

Quadro 2. Artigos selecionados para o estudo.

\begin{tabular}{|c|c|c|c|}
\hline Título & Ano & Periódico & Contexto \\
\hline $\begin{array}{l}\text { Conhecimento sobre câncer de mama entre } \\
\text { enfermeiros da atenção primária de Divinópolis/MG }\end{array}$ & 2020 & Revista Nursing & Educação \\
\hline $\begin{array}{l}\text { Conhecimento, atitude e prática de enfermeiros na } \\
\text { detecção do câncer de mama }\end{array}$ & 2020 & $\begin{array}{l}\text { Revista de } \\
\text { Enfermagem Escola } \\
\text { Anna Nery }\end{array}$ & Educação \\
\hline $\begin{array}{l}\text { Revisões da literatura científica: tipos, métodos e } \\
\text { aplicações em enfermagem }\end{array}$ & 2018 & $\begin{array}{l}\text { Revista portuguesa } \\
\text { de enfermagem de } \\
\text { reabilitação }\end{array}$ & Educação \\
\hline $\begin{array}{l}\text { Atuação do enfermeiro da atenção primária à saúde na } \\
\text { assistência oncológica }\end{array}$ & 2018 & $\begin{array}{l}\text { Revista Cogitare } \\
\text { Enfermagem }\end{array}$ & Cuidado \\
\hline $\begin{array}{l}\text { Aplicação do arco de Charlez } \\
\text { implementação de estratégias para } \\
\text { câncer de pênis }\end{array}$ & 2020 & REVISA & Educação \\
\hline $\begin{array}{l}\text { Gestão do cuidado à mulher na atenção primária: } \\
\text { estratégias para efetivação do processo de enfermagem }\end{array}$ & 2020 & $\begin{array}{l}\text { Enfermagem em } \\
\text { Foco }\end{array}$ & Cuidado \\
\hline $\begin{array}{l}\text { Detecção precoce do câncer de mama em Unidades } \\
\text { Básicas de Saúde }\end{array}$ & 2021 & $\begin{array}{l}\text { Acta Paulista de } \\
\text { Enfermagem }\end{array}$ & Cuidado \\
\hline $\begin{array}{l}\text { Ações públicas para o controle do câncer de mama no } \\
\text { Brasil: revisão integrativa }\end{array}$ & 2016 & $\begin{array}{l}\text { Revista Brasileira de } \\
\text { Enfermagem }\end{array}$ & Cuidado \\
\hline $\begin{array}{l}\text { Implantação da sistematização da assistência por } \\
\text { enfermeiras na atenção básica: facilidades e } \\
\text { dificuldades }\end{array}$ & 2021 & Jonah - UFPel & Cuidado \\
\hline $\begin{array}{l}\text { Actions for early detection of breast cancer in two } \\
\text { municipalities in the Western Amazon }\end{array}$ & 2021 & $\begin{array}{l}\text { Revista Brasileira de } \\
\text { Enfermagem }\end{array}$ & Cuidado \\
\hline $\begin{array}{l}\text { Tecnologia para educação em saúde na prevenção e } \\
\text { rastreamento do câncer de mama }\end{array}$ & 2021 & Revista Nursing & Educação \\
\hline $\begin{array}{l}\text { Prevenção e rastreamento de neoplasias femininas: } \\
\text { mama e colo do útero }\end{array}$ & 2018 & Acta Médica & Cuidado \\
\hline $\begin{array}{l}\text { Conhecimento de mulheres sobre fatores relacionados } \\
\text { ao câncer de mama }\end{array}$ & 2017 & $\begin{array}{lr}\text { Revista } & \text { De } \\
\text { Enfermagem } & \text { da } \\
\text { UFSM } & \end{array}$ & Educação \\
\hline Acesso ao exame de mamografia na atenção primária & 2017 & $\begin{array}{l}\text { Revista De } \\
\text { Enfermagem UFPE }\end{array}$ & Cuidado \\
\hline $\begin{array}{l}\text { Diretrizes para detecção precoce do câncer de mama } \\
\text { no Brasil. II - Novas recomendações nacionais, } \\
\text { principais evidências e controvérsias. }\end{array}$ & 2018 & $\begin{array}{l}\text { Cardeno de Saúde } \\
\text { Pública }\end{array}$ & Educação \\
\hline
\end{tabular}

Fonte: Autores (Brasília-DF, 2021).

O Câncer é uma das doenças crônicas não transmissíveis mais incidentes na população do Brasil e do mundo. Se excluirmos o Câncer de pele do tipo não melanoma, o Câncer de mama é o que possui a maior taxa de incidência em mulheres (INCA, 2020).

Ao longo dos anos as estratégias e programas governamentais que visam melhorar a qualidade dos atendimentos nas Unidades de Saúde e proporcionar um melhor prognóstico às mulheres já diagnosticadas vem sofrendo alterações que tem como objetivo abarcar de uma maneira mais humanizada e eficiente no que tange esse processo de prevenir/diagnosticar o Câncer de mama no Sistema Único de Saúde, temos como exemplo ao longo da história:

- Em 1984 foi o controle do câncer de mama no Brasil foi inserido no Programa de Assistência Integral à Saúde da Mulher, que tinha como premissa um cuidado mais amplo e em vários aspectos na saúde da mulher. 
- Em 1986 foi criado o Pro-Onco pelo Instituto Nacional do Câncer, mais tarde, em 1990 esse programa se tornou a Coordenação de Programas de Controle Câncer, que tinha como objetivo informar e educar sobre os tipos Neoplasia mais incidentes no Brasil, incluindo o Câncer de mama.

- No final dos anos 90 iniciou-se um movimento voltado à formulação de novas diretrizes e a sistematização da assistência voltada à detecção e precoce do Câncer de mama.

- Em 2004 teve o Consenso, que trouxe diretrizes técnicas para o controle desse tipo de Câncer no Brasil.

- Em 2005 efetivou-se o Programa Nacional de Atenção Oncológica, tendo como destaque o controle dos Cânceres de colo do útero e mama.

- Em 2006, foi incluindo como meta aos estados e municípios a melhoria dos indicadores que impactam na incidência de Câncer de mama através do Pacto pela Saúde.

- Países da Europa, Canada e Chile possuem modelos de atenção ao rastreamento bem-sucedidos e em abril de 2009 o INCA promoveu o Encontro Internacional Sobre Rastreamento do Câncer de Mama com o objetivo de melhorar o modelo assistencial da época.

- Em julho de 2009 foi implementado o SISMAMA (Sistema de informação do Câncer de Mama).

- Em maio de 2013, a política de atenção oncológica passou por uma atualização advinda da Política Nacional para a Prevenção e Controle do Câncer na Rede de Atenção à Saúde das Pessoas com Doenças Crônicas no âmbito do Sistema Único de Saúde (SUS) e no mesmo ano foi inserido o SISCAN (Sistema de Informação do Câncer), que abarcou o SISMAMA e SISCOLO (Sistema de Informação do Câncer de Colo do Útero).

- Através da portaria MS/GM 59 / 01 de out 2015, foram implementadas novas diretrizes para a detecção precoce do Câncer de mama dentro do SUS, seguindo um rigoroso processo de busca de evidências com o objetivo de amparar os profissionais da saúde em suas práticas clínicas, melhorando assim a qualidade da assistência (INCA, 2020).

Entre algumas leis que tangem essa questão do cuidado com o Câncer de mama se faz importante destacar a lei 11.664 de 2008 (Ministério da Saúde, 2008), conhecida como lei da mamografia, ela versa que o Sistema Único de Saúde deve garantir acesso à mamografia para todas as mulheres a partir dos 40 anos de idade. Outras leis que vieram para corroborar com esse movimento a favor da prevenção/detecção e tratamento precoce são a lei dos três dias (Ministério da Saúde, 2018) que permite ao trabalhador, seja homem ou mulher, se ausentar por até 3 dias a cada 12 meses para efetuar exames visando a detecção precoce de cânceres, a lei dos 60 dias (Ministério da Saúde, 2012) que afirma que o paciente diagnosticado com qualquer tipo de Câncer deve iniciar o tratamento pelo SUS em um prazo máximo de até 60 dias.

Nas Unidades Básicas de Saúde a atuação da enfermagem é de suma importância no que diz respeito à prevenção, detecção e diagnóstico precoce do Câncer de mama, porém essa assistência não fica limitada somente nesse nível de atenção, espera-se que a Atenção Primária na Saúde esteja em harmonia com os outros níveis de atenção, promovendo saúde desde o momento prevenção, diagnóstico, tratamento, reabilitação e cuidados paliativos dos usuários do Sistema Único de Saúde.

Sendo assim, fica em evidencia a importância da Atenção Primária na prevenção desse tipo de patologia, tendo em vista que ela e a porta de entrada dos usuários do sistema único de saúde. Entre as atividades voltadas para a prevenção e assistência que estão no escopo da enfermagem no âmbito da Atenção Básica de Saúde, podemos enfatizar ações educativas para a prevenção, acolhimento ao paciente recém diagnosticado, consulta de enfermagem, ações de rastreamento e acompanhamento da evolução da doença, encaminhamento para equipe multidisciplinar e atenção secundária/ terciária para realização de exames mais complexos, visitas domiciliares, quimioterapia e radioterapia, entre outras atividades englobadas no leque de opções que a enfermagem possui na Atenção Básica (Souza et al., 2018) 
A atenção primária atua com ações que visam a prevenção de agravos através da educação da população alvo intervindo em sua saúde levando em consideração seus fatores comportamentais e ambientais, essas ações além de prevenir o aparecimento de neoplasias, também confere maior longevidade aos pacientes, uma vez que estes estão adquirindo práticas de autocuidado.

Além da execução de suas atividades rotineiras na detecção precoce do Câncer de mama, o profissional enfermeiro deve sistematizar sua assistência de acordo com a realidade apresentada na comunidade em que está inserido, podendo obter assim um melhor resultado na prevenção dessa patologia, podendo também lançar mão de recursos educacionais visando aumentar a proximidade com a população alvo e lhes dando maior responsabilidade sobre seu estado de saúde (Moraes-Filho et al., 2020).

\subsection{Enfermagem na Atenção Primária à Saúde}

A Enfermagem é uma prática profissional essencial, é historicamente definida e faz parte de um contexto maior com o objetivo de gerar ações curativas por meio de conhecimentos específicos embasados cientificamente.

Compete ao Enfermeiro no âmbito da Atenção Primária à Saúde: Realizar consultas de enfermagem e procedimentos de acordo com protocolos estabelecidos por lei, bem como solicitar exames complementares, encaminhar a outros serviços quando necessário e prescrever medicações pertinentes à suas atividades dentro das Unidades Básicas de Saúde; Realizar atividades programadas e demanda espontânea; Planejar, gerenciar e avaliar as atividades desenvolvidas pelas Agentes Comunitários de Saúde; Realizar atividades de educação permanente da própria equipe de enfermagem e outros membros e participar do gerenciamento de insumos da unidade.

A Atenção Básica à Saúde (APS) é o primeiro nível de atenção e é caracterizada por um conjunto de ações em nível individual e coletivo, incluindo promoção e proteção da saúde, prevenção de doenças, diagnóstico, tratamento, restauração e manutenção da saúde, trabalhando de maneira integral visando impactar na situação atual da saúde e autonomia dos indivíduos e seus determinantes de saúde (Ministério da Saúde, 2011).

A Sistematização da Enfermagem (SAE) facilita o fluxo de trabalho do enfermeiro, aprimorando os processos de enfermagem (PE) com instruções e registros na evolução de enfermagem. Está associada à identidade do enfermeiro, traz muitas vantagens ao cuidado e deixa um amplo espaço para a autonomia profissional (Rosa et al, 2020).

Pela resolução 358/2009 do Conselho Federal de Enfermeiros (COFEN), SAE e a implantação do PE, composto por cinco etapas: Histórico de enfermagem, Diagnóstico de enfermagem, Planejamento de enfermagem, Implementação e Avaliação de enfermagem, presentes em qualquer ambiente, seja ele público ou privado, em que ocorra a prática da enfermagem profissional, devem ser realizados de forma ordenada e deliberada (COFEN, 2009).

\subsection{Prevenção do Câncer de mama em UBS}

Um dos grandes desafios da atualidade para o profissional Enfermeiro que atua na Atenção Básica à Saúde é compreender a mudança do perfil epidemiológico da atual sociedade e ao mesmo tempo inserir isso dentro de uma esfera maior, onde fatores como estilo de vida, envelhecimento, stress, obesidade contribuem diretamente para o aparecimento de doenças crônico-degenerativas.

Sendo assim, faz-se necessário que o profissional Enfermeiro atuante na UBS tenha ciência desse fato e atue com visão integral e solidez na prevenção do câncer de mama, para tal é preciso estar atualizado quanto aos métodos de deteç̧ão precoce e realizar ações de rastreamento na população adscrita (Ferreira et al., 2020).

O Ministério da Saúde se respalda em evidências científicas ao estabelecer as diretrizes para o controle do câncer de mama, para tanto, ações para educar e conscientizar a população são de suma importância para a prevenção dessa neoplasia. 
Visando intervir em fatores de risco à saúde da população, a Estratégia Saúde da Família assiste aos pacientes de maneira integral tendo um cuidado multiprofissional e é o modelo aplicado pelas Unidades Básicas de Saúde que por sua vez é a porta de entrada no SUS, sendo assim, faz-se necessário uma busca ativa de rastreamento através do exame de mamografia (MMG) com o intuito de identificar casos confirmados no início e potencializar o tratamento (Melo et al., 2021)

Na Atenção Primária o que se vê atualmente no que tange a prevenção do câncer de mama é o estímulo à população adscrita para se ter hábitos de vida saudáveis, controle da alimentação, praticar exercícios regularmente e controle da obesidade. Vale ressaltar que no nível de atenção secundário o enfermeiro treinado pode realizar o exame clínico das mamas com caráter preventivo, que se dá no momento do exame físico (Isabella et al., 2016).

\section{Considerações Finais}

A atuação da enfermagem no que diz respeito à prevenção do câncer de mama no escopo da Atenção Primária precisa ser incisivo e eficaz para se obter resultados efetivos e aumentar a expectativa de vida da população, para isso, é necessário que toda a equipe esteja em consonância e em direção das melhores ações de educação e conscientização, levando em consideração todos os fatores envolvidos nesse processo.

O estudo possibilitou um olhar mais panorâmico à um dos problemas de saúde públicas mais alarmantes da atualidade e traz à tona a importância de se respeitar todo o processo de enfermagem envolvido na prevenção, cura, reabilitação e cuidados paliativos no câncer de mama, porém para isso é preciso ter um fomento maior em todas as esferas do cuidado e por parte das políticas públicas de prevenção e cuidado de pacientes com câncer, tudo isso nos traz a reflexão de que é preciso acompanhar mais de perto o processo de trabalho do enfermeiro e realizar mais estudos envolvendo seu papel na prevenção do câncer de mama, tanto junto de equipe multidisciplinar, como quando atuando de maneira singular na Unidade Básica de Saúde.

\section{Referências}

Almeida, L., Santana, J., Silva, S., \& Melo, M. (2017). Acesso ao exame de mamografia na atenção primária. Revista de Enfermagem UFPE on line, 11(12), 4885-4894. https://doi.org/10.5205/1981-8963-v11i12a15023p4885-4894-2017

Barbosa, A. P., Ricacheneisky, L. F., \& Daudt, C. G. (2018). Prevenção e rastreamento de neoplasias femininas: mama e colo do útero. Acta Médica (Porto Alegre), 2(39), 335-345. https://ebooks.pucrs.br/edipucrs/acessolivre/periodicos/acta-medica/assets/edicoes/2018-2/arquivos/pdf/31.pdf

da Rosa, A., Zocche, D., \& Zanotelli, S. (2020). Gestão do cuidado à mulher na atenção primária: estratégias para efetivação do processo de enfermagem. Enfermagem em Foco, 11(1). https://doi.org/10.21675/2357-707X.2020.v11.n1.2670

Derenzo, N., Costa, M., Melo, W., Costa, C., Francisqueti, V., \& Bernuci, M. (2017). Conhecimento de mulheres sobre fatores relacionados ao câncer de mama. Revista de Enfermagem da UFSM, 7(3), 436 - 447. https://doi.org/10.5902/2179769225641

Ferreira, D. S., Bernardo, F. M., Costa, E. C., Maciel, N. S., Costa, R. L., \& Carvalho, C. M. (2020). Conhecimento, atitude e prática de enfermeiros na detecção do câncer de mama. Revista de Enfermagem Escola Anna Nery, 24(2). https://doi.org/10.1590/2177-9465-EAN-2019-0054

Filho, I. M., Francisco, C. R., Moraes, R. B., Félis, K. C., Filha, F. S., \& Sousa, T. V. (2020). Aplicação do arco de Charlez Maguerez na implementação de estratégias para prevenção do câncer de pênis: relato de experiência. REVISA, 9(4). https://doi.org/10.36239/revisa.v9.n4.p804a809

INCA, I. N. d. C. (2020, January 17). O que é Câncer. www.inca.gov.br. https://www.scielo.br/j/ean/a/fcH45Y8Q8HPfLqWFKKCmbMr/?lang=pt

INCA, I. N. d. C. (2020, May 12). Estimativa 2020. Casos estimados de câncer no Brasil. www.inca.gov.br. https://www.inca.gov.br/estimativa/introducao\#: :text=Para\%20o\%20Brasil\%2C\%20a\%20estimativa,c\%C3\%A2ncer\%20de\%20pele\%20n\%C3\%A3o\%20me lanoma.

INCA, M. d. S. (2021). Histórico das ações: controle do câncer de mama. www.inca.gov.br. https://www.inca.gov.br/controle-do-cancer-de-mama/historicodas-acoes.

Lei 11.664 de 2008, M. d. S. (2008). Lei da mamografia. www.planalto.gov.br. http://www.planalto.gov.br/ccivil_03/_ato2007-2010/2008/lei/111664.htm

Lei 12.732 de 2012, M. d. S. (2012). Lei dos sessenta dias. www.planalto.gov.br. http://www.planalto.gov.br/ccivil_03/_ato2011-2014/2012/lei/112732.htm

Lei 13.767 de 2018, M. d. S. (2018). Lei dos três dias. www.planalto.gov.br. http://www.planalto.gov.br/ccivil_03/_ato2015-2018/2018/lei/L13767.htm 
Research, Society and Development, v. 10, n. 16, e546101624057, 2021

(CC BY 4.0) | ISSN 2525-3409 | DOI: http://dx.doi.org/10.33448/rsd-v10i16.24057

Lopes Oliveira, D. A., Santos Dutra, C. R.., Santos Silva, M. E., Pereira de Oliveira, M. R., Queiroz de Lima, L. J., Pereira de Lima, A. S., \& Portela de Carvalho, F. (2021). Tecnologia para educação em saúde na prevenção e rastreamento do câncer de mama. Nursing (São Paulo), 24(275), 5530-5543. https://doi.org/10.36489/nursing.2021v24i275p5530-5543

Melo, F. B., Figueiredo, E. N., Panobianco, M. S., \& Rosa, A. S. (2021). Detecção precoce do câncer de mama em Unidades Básicas de Saúde. Acta Paulista de Enfermagem, 34. https://doi.org/DOI: 10.37689/acta-ape/2021AO02442

Ohl, I. C., Ohl, R. I., \& Goldman, R. E. (2016). Ações públicas para o controle do câncer de mama no Brasil: revisão integrativa. Revista Brasileira de Enfermagem. https://doi.org/10.1590/0034-7167.2016690424i

Portaria $\mathrm{N}^{\circ}$ 2.488, de 21 de outubro de 2011, M. d. S. (2011). Política Nacional de Atenção Básica. www.bvsms.saude.gov.br. https://bvsms.saude.gov.br/bvs/saudelegis/gm/2011/prt2488_21_10_2011.html

Resolução $\mathrm{n}^{\circ} 358$ de 15 de outubro de 2009, COFEN (2009). Sistematização da Assistência de Enfermagem. www.cofen.gov.br. http://www.cofen.gov.br/resoluo-cofen-3582009_4384.html

Santos, A. K., Sousa, M. S., Silva, A. F., Estrela, F. M., Lima, N. S., David, R. A., Sousa, T. J., \& Oliveira, D. F. (2021). Implantação da sistematização da assistência por enfermeiras na atenção básica: facilidades e dificuldades. JONAH, 11(2). https://doi.org/HTTPS://DOI.ORG/10.15210/JONAH.V11I2.20246

Santos, C. S., Araujo, A. C., Silva, F. M., Quadros, K. A., Santos, R. M., \& Andrade, S. N. (2020). Conhecimento sobre câncer de mama entre enfermeiros da atenção primária de Divinópolis/MG. Nursing, 23(267). https://doi.org/10.36489/nursing.2020v23i267p4452-4465

Silva, M. S., Gutiérrez, M. G., Figueiredo, E. N., Barbieri, M., Ramos, C. F., \& Gabrielloni, M. C. (2021). Actions for early detection of breast cancer in two municipalities in the Western Amazon. Revista Brasileira de Enfermagem, 2(74). https://doi.org/10.1590/0034-7167-2020-0165

Sousa, G. R., Cazola, L. H., \& Pícoli, R. P. (2018). Atuação do enfermeiro da atenção primária à saúde na assistência oncológica. Revista Cogitare Enfermagem, 23(4). https://doi.org/http://dx.doi.org/10.5380/ce.v23i4.58152

Sousa, L. M., Firmino, C. F., Marques-Vieira, C. M., Severino, S. S., \& Pestana, H. C. (2018). Revisões de literatura científica: Tipos, métodos e aplicações em Enfermagem. Revista Portuguesa de Enfermagem de Reabilitação, 1(1). https://doi.org/10.33194/rper.2018.v1.n1.07.4391

Migowski, A., Silva, G. A., Dias, M. B., Diz, M. P., Sant'ana, D. R., \& Nadanovsky, P. (2018). Diretrizes para detecção precoce do câncer de mama no Brasil. II - Novas recomendações nacionais, principais evidências e controvérsias. Caderno de Saúde Pública, 6(34). https://doi.org/10.1590/0102-311X00074817 